\title{
Bed Pedal Theory for Space Craft Using Grounded Approach: A Survey on Undergraduate Health Nutritionists in Lahore Pakistan
}

\author{
Umair Shahzad ${ }^{1,2,3,4}$ \\ ${ }^{1}$ Department of Computer Sciences, Virtual University of Pakistan, Lahore, Pakistan \\ ${ }^{2}$ Pakistan Aeronautics and Space Administration, Lahore, Pakistan \\ ${ }^{3}$ Department of Mass Communication Management, The Superior College Lahore, Lahore, Pakistan \\ ${ }^{4}$ Department of Mass Communication Forman Christian College (A Chartered University) Lahore, Lahore, Pakistan \\ Email address: \\ purpleresearchfeedback@gmail.com
}

To cite this article:

Umair Shahzad. Bed Pedal Theory for Space Craft Using Grounded Approach: A Survey on Undergraduate Health Nutritionists in Lahore Pakistan. International Journal of Astrophysics and Space Science. Vol. 7, No. 6, 2019, pp. 59-65. doi: 10.11648/j.ijass.20190706.11

Received: July 1, 2019; Accepted: November 9, 2019; Published: November 26, 2019

\begin{abstract}
The health of an astronaut is a major ingredient to accomplish a mission in space. Science has introduced many different inventions that are assisting mankind in many ways even in space. This research comprises of a grounded theory that has been further partially accepted by a survey on undergraduate health nutritionists for the protection of the health of an astronaut in space craft. When a human is in space there are many factors that can lead to muscular problems. To reduce the risk of muscular problems of legs in space a bed pedal theory for invention is presented to 200 undergraduate health nutritionists in Lahore Pakistan and their responses are taken for further inventing the bed pedal. The author has estimated that without any fatigue a person can utilize 150 calories by exercising with a bed pedal for approximately thirty minutes by taking very less area compared to a stationary bike even in small space crafts it can be easily attached to any place feasible for an astronaut to exercise. This research is based on quantitative research method to analyze a hypothesis that is built on the bed pedal invention theory by taking responses via closed-ended questionnaires for the results. The findings are analyzed by using descriptive statistics, correlation and t-test showing partial acceptance on the idea of this theory. The study has implications for the International Space Station Research and Development and Pakistan Aeronautics and Space Administration (if established) for future application.
\end{abstract}

Keywords: Bed Pedal Theory, Micro Gravity, Space Cycle, Space Exercise, Astronaut Health, Artificial Gravity, Kinetic Energy, Life Sciences

\section{Introduction}

In the environment of micro gravity weightlessness can be a major threat to the health of an astronaut. Instead of floating in the zero-gravity situation if any easily usable exercise tool introduced to the routine of an astronaut is provided that can assist the astronaut to break the aftereffects of the null gravity scenario it will be a milestone. For an Astronaut it is mandatory to maintain fitness in a prolonged spaceflight because there are more potential diseases and threats out there in space that can lead to serious loss including body weight etc. So one should have benefit from exercise. Astronauts lose bone and cardiovascular stress in space, and muscles atrophy because Earth's gravity is not acting on them through their everyday activities. Residents of the International Space Station exercise for about two hours per day in an attempt to stave off these diseases, but they still return to Earth very weak. As lifting weights in space is not useful, so the astronauts just use resistive exercises. The heart also weakens in space, sometimes causing astronauts to faint when they sit up or stand up quickly after their return to Earth [1].

A bike kind of centrifuge that creates artificial gravity may help astronauts combat muscle atrophy in space [2].

As a cycle is entirely dependent on the motion of a wheel so it becomes nearly impossible to ignore the significance of 
a wheel.

A wheel is defined as a circular frame of hard material that may be solid, partly solid and that is capable of turning on. According to the history, concept of wheel came out from a Sumerian (Erech) pictograph, dated about 3500 BC, shows a sledge equipped with wheels. The idea of wheeled transportation may have come from the use of logs for rollers, but the oldest known wheels were wooden disks consisting of three carved planks clamped together by transverse struts [3].

Wheel is such an invention if used in a specific direction may lead to required benefits. Where wheel is being used on earth in many of the mechanisms in the same way it is also being used in space. In space it is being used as part of different machinery, automobiles that run in outer space etc. So where shape as wheel is being used in space for many different purposes so it can also be used for the exercise of an astronaut. Some of the researches have also admitted that.

"Space Cycle is an artificial gravity exercise gym,"Caiozzo said. "The platform can be fitted with a treadmill; bike or any kind of exercise equipment and provides an environment for exercise under normal, Earthkind of similar loading conditions." [2].

Extensive isolation and integration efforts and resources will be required for actual spaceflight certification of a large rotating system such as the Space Cycle [4].

A habitat centrifuge will be essential for testing protocols and operations necessary to protect crews during long stays on the lunar or Martian surface [5].

Therefore, research on exercise machines specifically designed for space crafts is significant for the health of astronauts. In this research a bed pedal theory is introduced for the exercise of an astronaut in space.

\subsection{Objectives}

Below is the objective of this research.

This predictor research is conducted to determine whether a bed pedal can be a tool for protection of an astronaut's health in space crafts or not.

\subsection{Significance of the Study}

This research will be helpful in identifying the significance of bed pedal in space crafts on the health of an astronaut.

It can change the concept of scientists that a bed pedal can be a significant tool for an astronaut in space crafts and further they can investigate through proper experiments find a major exercise machine that can facilitate an astronaut's health.

\subsection{Grounded Theory}

This is a theory named "Bed Pedal Theory" that is being introduced or invented in this research article and this Bed pedal invention theory explains the Bed pedal and why it can be significant in a space craft for astronauts. A Bed pedal is defined as a combination of two wheels with having teeth shape on the edges mounted within a stand that have pedals on the center of both the sides of a single wheel, however the pedal wheel is partially attached to another wheel by tooth and when one wheel moves clockwise with the motion of a pedal the tooth of pedaling wheel forces other wheel to move in anticlockwise rotation this happens via kinetic energy, in this way a resistance pull is generated and the astronaut who does this exercise in space allows the body to remain in stable weight through legs exercise. However, there is an option provided in the bed pedal of holding weight according to astronaut strength so that more body specific workout can be conducted in a space craft. This invention is manual and does not require any kind of electrical source to run. It can be easily used by the force of a human being on the pedals of the "Bed pedal." It can be attached with a Bed that is why its name is introduced as a bed pedal. However, it can also be placed on the floor in front of a chair or any such place in a space craft where an astronaut can balance and move it through the legs

\subsection{Limitations}

This research is only limited to the responses taken on introducing an idea for the invention of a Bed pedal. Therefore, no experiment is conducted in order to analyze the physical results. As, space scenario is not present in Pakistan where this invention may occur.

\subsection{Hypothesis}

A hypothesis is assumed on the basis of which results are analyzed.

$\mathrm{H}_{1}$ Bed pedal can maintain health of an astronaut in prolonged space flight.

\subsection{Literature Review}

The previous researches conducted are few on cycleoriented exercise in space crafts. The researchers who have worked on such similar exercise machines have their own concepts and they have even tried to explain the benefits of such exercise. One out of which is "Self-Powered Human Centrifuge, or Space Cycle." This proposed research explained the use of space cycle for prevention from harmful physiologic effects of prolonged human exposure to space flight's microgravity [6].

Through a study at the University of California, Irvine, the National Space Biomedical Research Institute (NSBRI) is exploring the concept of a Space Cycle for inflight resistance-training exercise [2].

Space Cycle is an artificial gravity exercise gym that enables the rider to perform resistance-training exercises without the use of weights. To achieve the desired amount of force, the rider on the left powers the cycle while the rider on the right performs squats [7].

If this grounded theory will be experimented by the Astronauts in space then it is possible that they may soon have another weapon in the fight against the muscle-wasting effects of living in space. And it will be low-tech, a cyclepowered centrifuge that creates its own gravity [8].

In past many researchers have put forward their concepts 
and some of them even had experimented in earth's environment one of the very popular research that has been conducted in past has been cited in different articles and their crux is mentioned. The contraption, called the Space Cycle, spins to create a force that mimics the pull of gravity. The device consists of a central spindle with a pair of attached harnesses, one of which has pedals that drive the machine's rotation [8].

Pedaling a bicycle in space could one day make astronauts in space feel as if they are on Earth again. More importantly, it might be a way to prevent the bone and muscle loss that plagues astronauts on long space missions, say scientists developing a human-powered system to create Earth-kind of similar gravity conditions [1].

Bed pedal can be a protective exercise machine for astronauts in space craft [9].

The motion generates pressure on the rider, forcing him against the seat in a manner similar to the effect of gravity on Earth. On the platform, the other person performs squat exercises. Instruments on the device report the separate work rates of the participants [7].

Kinetic energy is a form of energy that an object or a particle has by reason of its motion. If work, which transfers energy, is done on an object by applying a net force, the object speeds up and thereby gains kinetic energy. Kinetic energy is a property of a moving object or particle and depends not only on its motion but also on its mass. The kind of motion may be translation (or motion along a path from one place to another), rotation about an axis, vibration, or any combination of motions. [10]

Translational kinetic energy of a body is equal to one-half the product of its mass, $\mathrm{m}$, and the square of its velocity, $\mathrm{v}$, or $1 / 2 \mathrm{mv}^{2}$. This formula is valid only for low to relatively high speeds; for extremely high-speed particles it yields values that are too small. When the speed of an object approaches that of light $(3 \times 108$ meters per second, or 186,000 miles per second), its mass increases, and the laws of relativity must be used. Relativistic kinetic energy is equal to the increase in the mass of a particle over that which it has at a point multiplied by the square of the speed of light [10].

The unit of energy in the meter-kilogram-second system is the joule. A two-kilogram mass (something weighing 4.4 pounds on Earth) moving at a speed of one meter per second (slightly more than two miles per hour) has a kinetic energy of one joule. In the centimeter-gram-second system the unit of energy is the erg, 10-7 joule, equivalent to the kinetic energy of a mosquito in flight. Other units of energy also are used, in specific contexts, such as the still smaller unit, the electron volt, on the atomic and subatomic scale [10].

For a rotating body, the moment of inertia, I, corresponds to mass, and the angular velocity (omega), $\omega$, corresponds to linear, or translational, velocity. Accordingly, rotational kinetic energy is equal to one-half the product of the moment of inertia and the square of the angular velocity, or $1 / 2 \mathrm{I} \omega^{2}[10]$.

The total kinetic energy of a body or a system is equal to the sum of the kinetic energies resulting from each type of motion.

AIR RESISTANCE: Air resistance is similar to friction. It is caused by the molecules in the air pushing against a craft in flight. It is what slows parachutists down and keeps them from crashing into the Earth. There is no air resistance in space because there's no air in space [11].

FRICTION: When you hit the brakes in a car, the wheels slow down and friction between the road and the wheels slows the entire car. Friction, caused by things in physical contact with each other, is largely absent in space. Space is almost totally empty, so there is no "road" for the space ship to rub against [11].

\section{Methodology}

In this research grounded theory approach is used because the theory is not adopted from any other research. This theory is directly invented in this research article. Quantitative research technique is used to further have responses on the theory and results are analyzed using SPSS for the investigation of the hypothesis.

\subsection{Quantitative Research Questionnaire}

This research is based on quantitative research method where "Bed Pedal Invention Scale" as closed-ended questionnaire is used. Numbers of questions in the questionnaire are six that are asked from each respondent during the survey. These questions are based on the hypothesis of the research on the basis of which the respondents have filled the questionnaire after viewing the Bed pedal's image. The questionnaire is of paper and pencil type. The responses are taken on the basis of available sampling technique from two different universities of Lahore Pakistan. Likert type scale with five-point is used as a response format in the questionnaire.

Likert is a proper scale, which rises up out of aggregate reactions to an arrangement of things. Likert type scale is defined as a scale, which can extend from strongly disagree to strongly agree [12].

A Likert scale is a survey scale that represents a set of answer options either numeric or verbal. It covers a range of opinions on a topic. It's always part of a closed-ended question (a question that presents respondents with prepopulated answer choices. The Likert scale falls under the definition of a survey scale, in which five or seven-point scale is involved [13].

Likert scale is significant in this study because as compare to social sciences the neutral value is required in this research to provide detailed analysis of the responses. For instance, it is possible that bed pedal might not have any kind of effect on an astronaut's health so this means if a question is asked regarding difference in exercising or not the possible answer might be neutral.

\subsection{Participants and Data Collection}

To check the responses on the idea for the invention of this theory units of analysis are the undergraduate health nutritionists. An image is shown to 200 undergraduate health 
nutritionists belonging to two different universities from the departments related to health and nutrition in Lahore Pakistan.

If "Bed Pedal" is an object that has wheel A and wheel B connected together where wheel $\mathrm{A}$ is a driver wheel and wheel $\mathrm{B}$ is a driven wheel then if a force of 1 newton is transmitted to the pedal downwards then wheel A will move clockwise whereas, wheel B will move by the force applied by wheel A through pedal and wheel $\mathrm{B}$ will move anticlockwise.

Figure 1 of the bed pedal in the scenario is introduced in front of the students and then they are asked to provide their feedback by filing the "Bed Pedal Invention Scale." The responses of the students are recorded using "Statistical Package for the Social Sciences." On the basis of which results are carried out and conclusion is drawn.

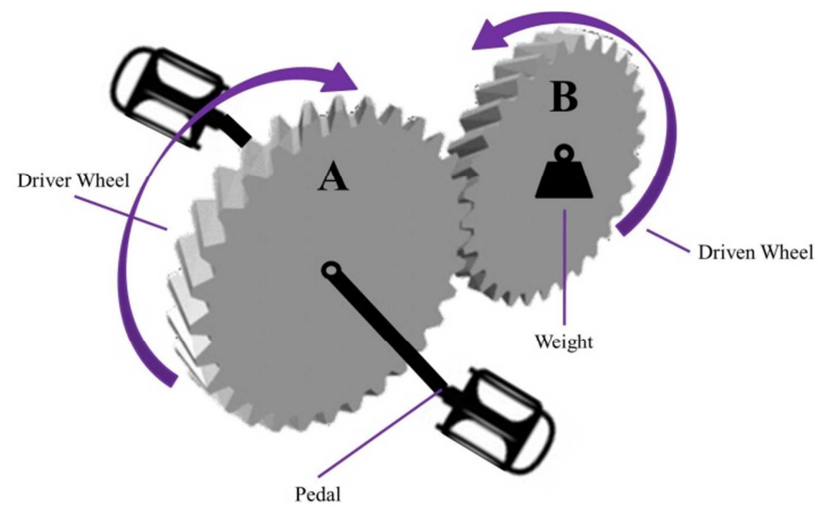

Figure 1. Bed pedal invention design.

The quantity of sample size out of the population of undergraduate health nutritionists is selected on the basis of a website (http://www.surveysystem.com/sscalc.htm) that automatically generates the quantity of the sample size. Here according to this scenario two universities are visited for the responses "University Institute of Diet \& Nutritional Sciences-The University of Lahore" and "University of
Health Sciences, Lahore." First the respondents are shown the Bed pedal image and after that respondents are asked to analyze this invention as a protective tool for the astronauts in protection of their health in space crafts. Health nutritionists are selected for the responses because they know that if a person is in a very restricted condition what can possibly suit that person for health stability in eating and exercise.

\section{Results and Findings}

The findings show that mainly bed pedal can be invented for health protection of astronauts in space crafts. It has significance towards the routine exercise of an astronaut that can lead to the restoration of health in positive ways but the results are broad and require more research to further investigate so that astronaut health conditions can be much improved in future. The respondents answered the questions of bed pedal invention scale according to that the results are received and are further mentioned. Hence, $\mathrm{H}_{1}$ is partially accepted in this research, whereas the respondents still have doubts that bed pedal will protect the range motion of ankles and knees in space crafts. Collecting response is a very difficult task. Usually the researchers appoint volunteers or hire third party individuals or companies for data collection but here the researcher himself has collected the data for the findings of the research. The researcher is able to collect the responses from 200 students in this research. However, the six questions asked in from the respondents are showing a wide different in the selection of the responses because as it is not so clear either this will work in space or not so that is why the difference is very much. First, the descriptive statistics are shown and then correlation and independent sample t-test is also conducted in this research to see what more can be concluded in this research Table 1 shows the details of the results:

Table 1. Descriptive Results and Findings of Bed Pedal Invention Scale.

\begin{tabular}{|c|c|c|c|c|c|c|c|c|c|c|}
\hline \multirow{2}{*}{ Sr. } & \multirow{2}{*}{ Questions } & \multicolumn{5}{|c|}{ Undergraduate Health Nutritionists Response \% of Total $\mathbf{N}$} & \multirow[b]{2}{*}{$\mathbf{x} \sqcup$} & \multirow{2}{*}{$\begin{array}{l}\mathbf{x} \\
\square\end{array}$} & \multirow{2}{*}{ Mo } & \multirow{2}{*}{ SD } \\
\hline & & $\begin{array}{l}\text { Strongly Disagree } \\
\end{array}$ & Disagree & Neutral & Agree & Strongly Agree & & & & \\
\hline 1 & $\begin{array}{l}\text { A Bed pedal can be invented for exercising } \\
\text { in space crafts. }\end{array}$ & 5 & 10 & 39 & 39 & 7 & 3.33 & 3 & 3 & .930 \\
\hline 2 & $\begin{array}{l}\text { In the scenario of micro gravity a bed pedal } \\
\text { can be a significant tool for reducing legs } \\
\text { oriented muscular problems. }\end{array}$ & 8 & 9.5 & 31.5 & 34 & 17 & 3.43 & 4 & 4 & 1.123 \\
\hline 3 & $\begin{array}{l}\text { A Bed pedal can be a less area consuming } \\
\text { exercise machine in space crafts. }\end{array}$ & 3.5 & 7 & 39 & 44.5 & 6 & 3.43 & 4 & 4 & .847 \\
\hline 4 & $\begin{array}{l}\text { A Bed pedal can be a way to utilize } \\
\text { approximately } 150 \text { calories within half an } \\
\text { hour. }\end{array}$ & 2 & 25.5 & 29 & 32 & 11.5 & 3.26 & 3 & 4 & 1.027 \\
\hline 5 & $\begin{array}{l}\text { A Bed pedal can be a way to protect the } \\
\text { range motion of ankles and knees in space } \\
\text { crafts. }\end{array}$ & 7.5 & 40 & 17 & 23.5 & 11.5 & 3.18 & 3 & 2 & 3.868 \\
\hline 6 & $\begin{array}{l}\text { A Bed pedal can be a low risk exercise } \\
\text { machine for stress and injuries in space } \\
\text { crafts. }\end{array}$ & 1 & 6.5 & 31 & 35 & 26.5 & 3.80 & 4 & 4 & .942 \\
\hline
\end{tabular}


calories within 30 minutes work out [14]. So it is estimated that a bed pedal can utilize calories up to half of it. Remaining results are based on the responses.

\subsection{Correlation}

Here the person correlation is used to see relationship between the variables of bed pedal exercise for astronaut's health. Pearson correlation coefficients (r) can take on only values from -1 to +1 [15].

The sign out the front indicates whether there is a positive correlation (as one variable increases, so too does the other) or a negative correlation (as one variable increases, the other decreases). The size of the absolute value (ignoring the sign) provides an indication of the strength of the relationship. A perfect correlation of 1 or -1 indicates that the value of one variable can be determined exactly by knowing the value on the other variable [12].

So in this research Pearson correlation shows that there is both positive and negative correlation between the items of bed pedal exercise for astronaut's health as mentioned in Table 2. Therefore, the items of bed pedal exercise for astronaut's health have the accuracy in the Bed Pedal Invention Scale and precision in the responses taken.

Table 2 Astronaut's Health from Bed Pedal Exercise Correlation; Shows correlation between items of Bed Pedal Exercise.

Table 2. Correlation between items of Bed Pedal Exercise.

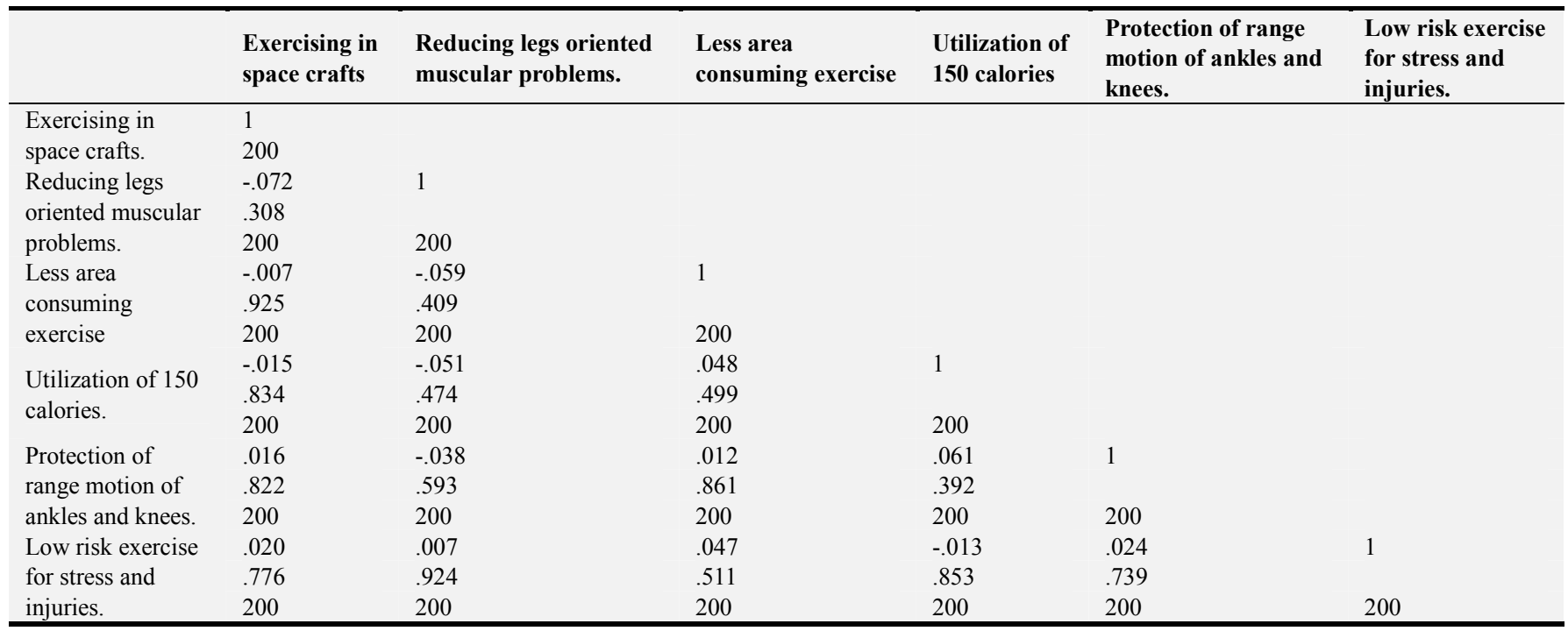

Pearson Correlation Sig. (2-tailed) N.

Table 2 shows that there is a positive correlation between exercising in space crafts and protection of range motion of ankles and knees $(\mathrm{r}=.016, \mathrm{n}=200, \mathrm{p}=.822)$, exercising in space crafts and low risk exercise for stress and injuries $(\mathrm{r}=.020, \mathrm{n}=200, \mathrm{p}=.776)$. Whereas, the table also shows negative correlation between exercising in space crafts and reducing legs oriented muscular problems $(\mathrm{r}=-.072, \mathrm{n}=200$, $\mathrm{p}=.308$ ), exercising in space crafts and less area consuming exercise $(\mathrm{r}=-.007, \mathrm{n}=200, \mathrm{p}=.925)$, exercising in space crafts and utilization of 150 calories $(r=-.015, n=200, p=.834)$.

Table 2 shows that there is a positive correlation between reducing legs oriented muscular problems and low risk exercise for stress and injuries $(r=.007, n=200, p=.924)$, whereas, the table also shows negative correlation between reducing legs oriented muscular problems and exercise in space $(\mathrm{r}=-.072, \mathrm{n}=200, \mathrm{p}=.308)$, reducing legs oriented muscular problems and less area consuming exercise $(\mathrm{r}=-.059$, $\mathrm{n}=200, \mathrm{p}=.409$ ), reducing legs oriented muscular problems and utilization of 150 calories $(\mathrm{r}=-.051, \mathrm{n}=200, \mathrm{p}=.474)$, reducing legs oriented muscular problems and protection of range motion of ankles and knees $(r=-.038, n=200, p=.593)$.

Table 2 shows that there is a positive correlation between less area consuming exercise and utilization of 150 calories $(\mathrm{r}=.048, \mathrm{n}=200, \mathrm{p}=.499)$, less area consuming exercise and low risk exercise for stress and injuries $(r=.047, n=200$, $\mathrm{p}=.511$ ). Whereas, the table also shows negative correlation between less area consuming exercise and exercising in space crafts $(\mathrm{r}=-.007, \mathrm{n}=200, \mathrm{p}=.925)$, less area consuming exercise and reducing legs oriented muscular problems $(\mathrm{r}=-.059$, $\mathrm{n}=200, \mathrm{p}=.409$ ), less area consuming exercise and protection of range motion of ankles and knees $(r=-.038, n=200$, $\mathrm{p}=.593)$.

Table 2 shows that there is a positive correlation between Utilization of 150 calories and Less area consuming exercise $(\mathrm{r}=.048, \mathrm{n}=200, \mathrm{p}=.499)$, Utilization of 150 calories and Protection of range motion of ankles and knees $(r=.061$, $\mathrm{n}=200, \mathrm{p}=.392$ ). Whereas, the table also shows negative correlation between Utilization of 150 calories and Exercising in space crafts $(\mathrm{r}=-.015, \mathrm{n}=200, \mathrm{p}=.834)$, Utilization of 150 calories and Reducing legs oriented muscular problems $(\mathrm{r}=-.051, \mathrm{n}=200, \mathrm{p}=.474)$, Utilization of 150 calories and Low risk exercise for stress and injuries. $(\mathrm{r}=-.013, \mathrm{n}=200, \mathrm{p}=.853)$.

Table 2 shows that there is a positive correlation between protection of range motion of ankles and knees and exercising in space crafts $(\mathrm{r}=.016, \mathrm{n}=200, \mathrm{p}=.822)$, protection 
of range motion of ankles and knees and low risk exercise for stress and injuries $(\mathrm{r}=.024, \mathrm{n}=200, \mathrm{p}=.739)$, protection of range motion of ankles and knees and utilization of 150 calories $(\mathrm{r}=.061, \mathrm{n}=200, \mathrm{p}=.392)$. whereas, the table also shows negative correlation between protection of range motion of ankles and knees and reducing legs oriented muscular problems $(\mathrm{r}=-.038, \mathrm{n}=200, \mathrm{p}=.593)$, protection of range motion of ankles and knees and less area consuming exercise $(\mathrm{r}=-.038, \mathrm{n}=200, \mathrm{p}=.593)$.

Table 2 shows that there is a positive correlation between low risk exercise for stress and injuries exercising in space crafts and $(\mathrm{r}=.020, \mathrm{n}=200, \mathrm{p}=.776)$, low risk exercise for stress and injuries and reducing legs oriented muscular problems $(\mathrm{r}=.007, \mathrm{n}=200, \mathrm{p}=.924)$, low risk exercise for stress and injuries and less area consuming exercise $(r=.047$, $\mathrm{n}=200, \mathrm{p}=.511)$, protection of range motion of ankles and knees and low risk exercise for stress and injuries $(\mathrm{r}=.024$, $\mathrm{n}=200, \mathrm{p}=.739$ ). Whereas, the table also shows negative correlation between low risk exercise for stress and injuries and utilization of 150 calories $(r=-.013, n=200, p=.853)$.

\subsection{Independent Sample T Test}

Here the independent-samples t-test is used to see the difference in the responses of the items of Bed Pedal Exercise for Astronaut's Health among gender.

An independent-samples t-test is used when you want to compare the mean score, on some continuous variable, for two different groups of subjects [12].

So in this research independent-samples t-test shows that there is no statistically significant difference between the responses of bed pedal exercise for astronaut's health by gender as mentioned in Table 3. Therefore, the responses of bed pedal exercise for astronaut's health have the accuracy in the Bed Pedal Invention Scale and precision in the responses taken.

Table 3 Gender difference in Variable of Bed Pedal Exercise for Astronaut's Health; Show difference between a Variable Bed Pedal Exercise for Astronaut's Health by Gender.

Table 3. Gender difference Independent Sample T Test.

\begin{tabular}{llllllll}
\hline Dependent Variable & Gender & N & Mean & Std. Deviation & t & df & Sig. (2-tailed) \\
\hline Bed Pedal Exercise for & Female & 67 & 20.5373 & 6.89165 & \multirow{2}{*}{296} & \multirow{2}{*}{198} & .767 \\
Astronaut's Health & Male & 133 & 20.3383 & 2.53726 & & \\
\hline
\end{tabular}

According to table 3 an independent samples t-test is conducted to compare the bed pedal exercise for astronaut's health scores for males and females. There is no significant difference in scores for males $(\mathrm{M}=20.3383, \mathrm{SD}=2.53726)$ and females $\mathrm{M}=20.5373, \mathrm{SD}=6.89165 ; \mathrm{t}$ (200) $=.296 .767$ (two tailed).

\section{Discussion}

As mentioned in introduction, the purpose of this study is to analyze the responses from the sample of the target audience and view either health nutritionist undergraduate students accept this concept or not. 200 students participated in the study by completing single closed-ended questionnaire survey. The primary limitation of this study is convenient sampling technique, small sample size out of the target population and lack of situation to experimentally test this bed pedal theory concept. One more limitation of this study is few specific academic institute located in Lahore, Pakistan because according to the scenario of this research two specific institutes were selected that had health nutritionists programs where physical and actual health related care knowledge is wide enough to have responses. In the present study, it is outside the scope to control other variables that might had contributed to an increase or decrease in the acceptance of the bed pedal concept. It is significant to note that bed pedal invention will be a few of many tools that will be used in future by astronauts in space. Therefore, the conclusions of this study should be viewed within the broader context of the theoretical partial acceptance of bed pedal of undergraduate graduate students. The conclusions of this study contribute to the literature detailing the role bed pedal exercise in space crafts in the lives of astronauts.

\section{Conclusion}

According to this study bed pedal can be a protective exercise machine for astronauts in space crafts. This partially proves that the idea of Bed pedal invention theory is accepted in the setting of Lahore Pakistan towards undergraduate health nutritionists but the range motion of ankles and knees may not be affected, but as this is a muscular exercise so it is definite that muscular protection will be provided by this exercise. However, calories will also be utilized by the astronaut to properly digest the intake of food in space. Even this will not require a large amount of space to fit in and in less occupancy the bed pedal will be used. This will possibly be a low risk exercise machine because of not having a complicated and wide structure there will be less chances of injuries or accidents while using it in the space craft. Overall the exercise mechanism is a potential way for health and requires attention. Further "Bed Pedal Invention Response Model" shows the use of Bed pedal for health protection of astronauts in space crafts. 


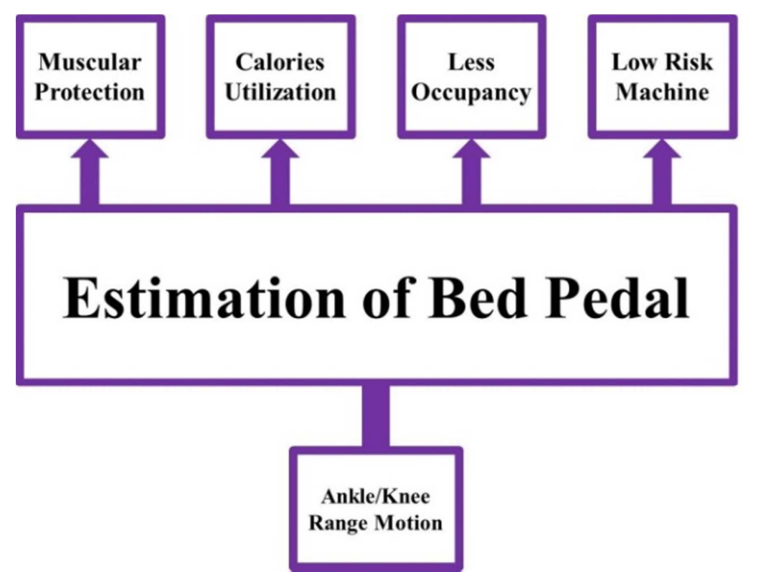

Figure 2. Bed Pedal Invention Response model.

It is an essence of a human to keep searching for something new. This theory can be further modified and improved by researchers in future who require it to study in any of the ways. The $\mathrm{H}_{5}$ rejection can be analyzed and new ways of protecting range motion of ankles and knees in space crafts can be invented even if the micro gravity does not allow so but there are still doors open for new inventions. Even if any such research conducted for a good cause can bring positive changes to the relevant field. Life sciences research is very necessary for country to work on because it is the foundation of a healthy life.

\section{Dedication and Acknowledgements}

This research article is dedicated to a peaceful country Pakistan. May peace prevail in Jammu and Kashmir an area of Pakistan! There is nothing better than love that can lead towards the upbringing of a society. The blood that is being wasted of Kashmiris is precious and one day every Kashmiri will live the live life with pride as a Pakistani. It is the knowledge that brings awareness in a society. This research article is generated with the satisfactory efforts of the student Umair Shahzad and those who were involved in this research including respondents etc. This quantitative research is written for academic cause and it is for awareness among researchers so that further more researchers can publish researches in the world.

\section{References}

[1] Young, K., Space Cycle may boost astronauts' fitness. New scientist, 2005.

[2] National Space Biomedical Research Institute, Space Cycle Tests Artificial Gravity As Solution To Muscle Loss, in ScienceDaily. 2005.

[3] The Editors of Encyclopaedia Britannica. Wheel. 2019 [cited 2019 May 17]; Available from: https://www.britannica.com/technology/wheel.

[4] Kreitenberg, A., J. Witmer, and J. Spiegel, Space cycle Ground-based prototype development. 2000.

[5] Young, L., K. Yajima, and W. Paloski, Artificial gravity research to enable human space exploration. Paris: International Academy of Astronautics, 2009: p. 1-37.

[6] Kreitenberg, A., et al., The" Space Cycle" Self Powered Human Centrifuge: a proposed countermeasure for prolonged human spaceflight. Aviation, space, and environmental medicine, 1998.69 (1): p. 66-72.

[7] MEDICA. Space Cycle Prevents Astronauts from Muscle Loss. MEDICA 2019 [cited 2019 August 27]; Available from: https://www.medicatradefair.com/en/News/Archive/Space_Cycle_Prevents_Astro nauts_from_Muscle_Loss.

[8] Hopkin, M., Space cycle makes artificial gravity. nature, 2005.

[9] Shahzad, U. Bed Paddle in Space Crafts: A Grounded Theory Using Survey Technique on Undergraduate Health Nutritionists in Lahore, Pakistan. in 6th International Space Station Research and Development Conference. 2017. Washington, District of Columbia: American Astronautical Society Center for the Advancement of Science in Space National Aeronautics and Space Administration.

[10] Encyclopædia Britannica. Kinetic energy. 2017 [cited 2019 August 31]; Available from: https://www.britannica.com/science/kinetic-energy.

[11] Qualitative Reasoning Group. Is there any resistance to movement in space for DS1? Propulsion 1990 [cited 20196 July]; Available from: www.qrg.northwestern.edu/projects/vss/docs/propulsion/2resisting-movement-in-space.html.

[12] Pallant, J., SPSS survival manual: a step by step guide to data analysis using SPSS for Windows. 2004, Buckingham: Open University Press.

[13] Survey Monkey. Learn when and how to use Likert scale survey questions. 2019 [cited 2019 October 13]; Available from: https://www.surveymonkey.com/mp/likert-scale/.

[14] Dilthey, M.R. How Many Calories Are Burned on a Stationary Bike in 30 Minutes? 2016 [cited 201720 June]; Available from: http://www.livestrong.com/article/217361-musclestationary-bike-work/.

[15] The Editors of Encyclopaedia Britannica. Correlation. 2011 [cited 2019 November 16]; Available from: https://www.britannica.com/science/correlation.

\section{Biography}

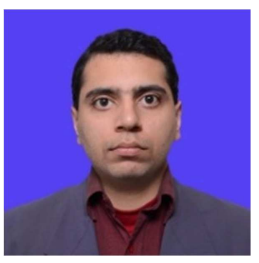

Umair Shahzad, He did his Master of Computer Science from Virtual University of Pakistan. He has many distance learning computer science related certifications from Foreign Institutions. He did his M. Phil. Mass Communication Management from The Superior College Lahore. He did his graduation in B. A. (Hons.) major in Mass Communication from Forman Christian College (A Chartered University) Lahore, Punjab, Pakistan. His major research affiliations are mass communication, education, computer science, health, STEM, universe, peace, academics and life science. The researcher works for peace in Pakistan and wants to stop brutality in Jammu and Kashmir by India. 